Supplement of Geosci. Model Dev., 8, 3545-3561, 2015

http://www.geosci-model-dev.net/8/3545/2015/

doi:10.5194/gmd-8-3545-2015-supplement

(C) Author(s) 2015. CC Attribution 3.0 License.

(c) (i)

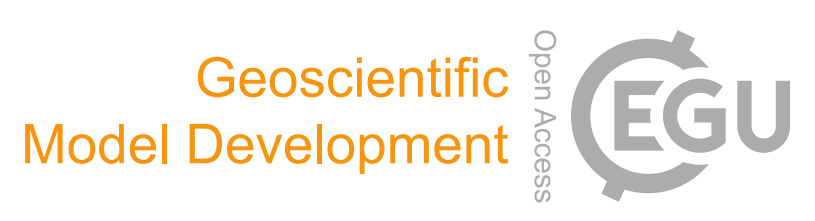

Supplement of

\title{
Modelling Mediterranean agro-ecosystems by including agricultural trees in the LPJmL model
}

M. Fader et al.

Correspondence to: M. Fader (marianela.fader@imbe.fr)

The copyright of individual parts of the supplement might differ from the CC-BY 3.0 licence. 
Table S1: Determination of harvested areas for land use dataset compilation. The stars indicate crops that are implemented in this study.

\begin{tabular}{|c|c|c|}
\hline LPJmL crops & $\begin{array}{l}\text { Data source for } \\
\text { harvested area }\end{array}$ & Crops in source \\
\hline Citrus $^{*}$ & Portmann et al. 2011 & Citrus \\
\hline Cotton* & Portmann et al. 2011 & Cotton \\
\hline Date Palm* & Portmann et al. 2011 & Date palm \\
\hline Fodder grass* & Portmann et al. 2011 & Fodder grasses \\
\hline Grapes* & Portmann et al. 2011 & Grapes / Vine \\
\hline Groundnuts & Portmann et al. 2011 & Groundnuts / peanuts \\
\hline Maize & Portmann et al. 2011 & Maize \\
\hline $\begin{array}{l}\text { Manage } \\
\text { Grasslands }\end{array}$ & $\begin{array}{l}\text { Ramankutty et al. } \\
2008\end{array}$ & Grasslands \\
\hline $\begin{array}{l}\text { Non Citrus } \\
\text { Orchards* }\end{array}$ & Monfreda et al. 2008 & $\begin{array}{l}\text { Apples, Apricots, Avocados, Bananas, Carobs, Cashew apple, } \\
\text { Cherries, Figs, Fruit fresh (other), Fruit tropical fresh (other), } \\
\text { kiwi, Mangoes, Papayas, Peaches and nectarines, Pears, } \\
\text { Persimmons, Plums, Quinces, Sour, cherries, Stone fruit } \\
\text { (other) }\end{array}$ \\
\hline Nuts Trees* & Monfreda et al. 2008 & $\begin{array}{l}\text { Almonds, Brazil Nuts, Cashew nuts, Chestnuts, Hazelnuts, } \\
\text { Karite nuts, Kola nuts, Nuts (other), Pistachios, Walnuts }\end{array}$ \\
\hline Olives* $^{*}$ & Monfreda et al. 2008 & Olives \\
\hline Others & Portmann et al. 2011 & Oil palm, Cocoa, Coffee, Others perennial, Others annual \\
\hline Potatoes* & Portmann et al. 2011 & Potatoes \\
\hline Pulses & Portmann et al. 2011 & pulses \\
\hline Rapeseed & Portmann et al. 2011 & Rapeseed / canola \\
\hline Rice & Portmann et al. 2011 & Rice \\
\hline Soybeans & Portmann et al. 2011 & Soybeans \\
\hline Sugar Cane & Portmann et al. 2011 & Sugar cane \\
\hline Sunflower & Portmann et al. 2011 & Sunflower \\
\hline $\begin{array}{l}\text { Temperate } \\
\text { Cereals }\end{array}$ & Portmann et al. 2011 & Wheat, barley, rye \\
\hline Temperate Roots & Portmann et al. 2011 & Sugar beet \\
\hline Tropical Cereals & Portmann et al. 2011 & Millet, sorghum \\
\hline Tropical Roots & Portmann et al. 2011 & Cassava \\
\hline Vegetables* & Monfreda et al. 2008 & $\begin{array}{l}\text { Artichokes, Asparagus, Cabbages, Carrots, Cauliflower, } \\
\text { Chillies and peppers (green), Cucumbers and gherkins, } \\
\text { Eggplants, Garlic, Green Beans or Beans (green), Broadbeans } \\
\text { (green), Green Corn, Onions and shallots (green), Peas } \\
\text { (green), Lettuce, Cantaloupes and other melons, Mushrooms, } \\
\text { Okra, Onions (dry), Pumpkins squash (gourds), Spinach, } \\
\text { String Beans, Tomatoes, Vegetables fresh (other), } \\
\text { Watermelons }\end{array}$ \\
\hline
\end{tabular}


1

2

3

4

5

6

7

8

9

10

11

12

13

14

15

16

Maximal monthly harvested areas for 26 irrigated and rainfed crops for year 2000 (Portmann et al. 2011, MIRCA)

Fractions of cropland and pasture for year 2000 (Ramankutty et al., 2008, RAM Harvested areas for 24 crops

Decadal fractional area of cropland and pasture per cell for 1700-2005 (Klein Goldewijk et al. 2011, HYDE)

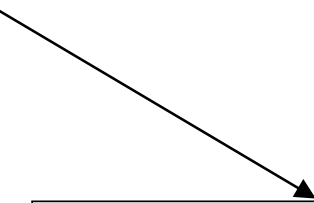

$M M R=$ Land-use map for 2000. Physical cultivation areas of 24 crops (irrigated and rainfed)

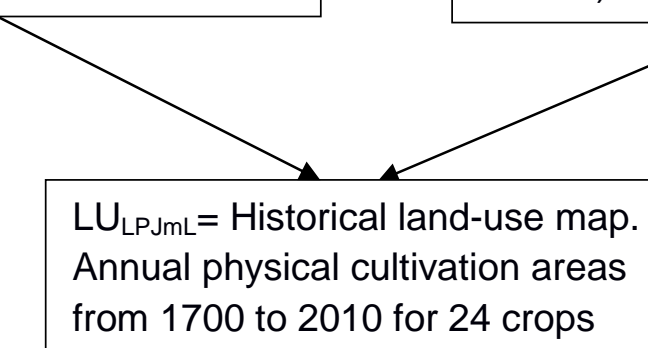

Fig. S1: Compilation of the land use dataset used as input for LPJmL. 

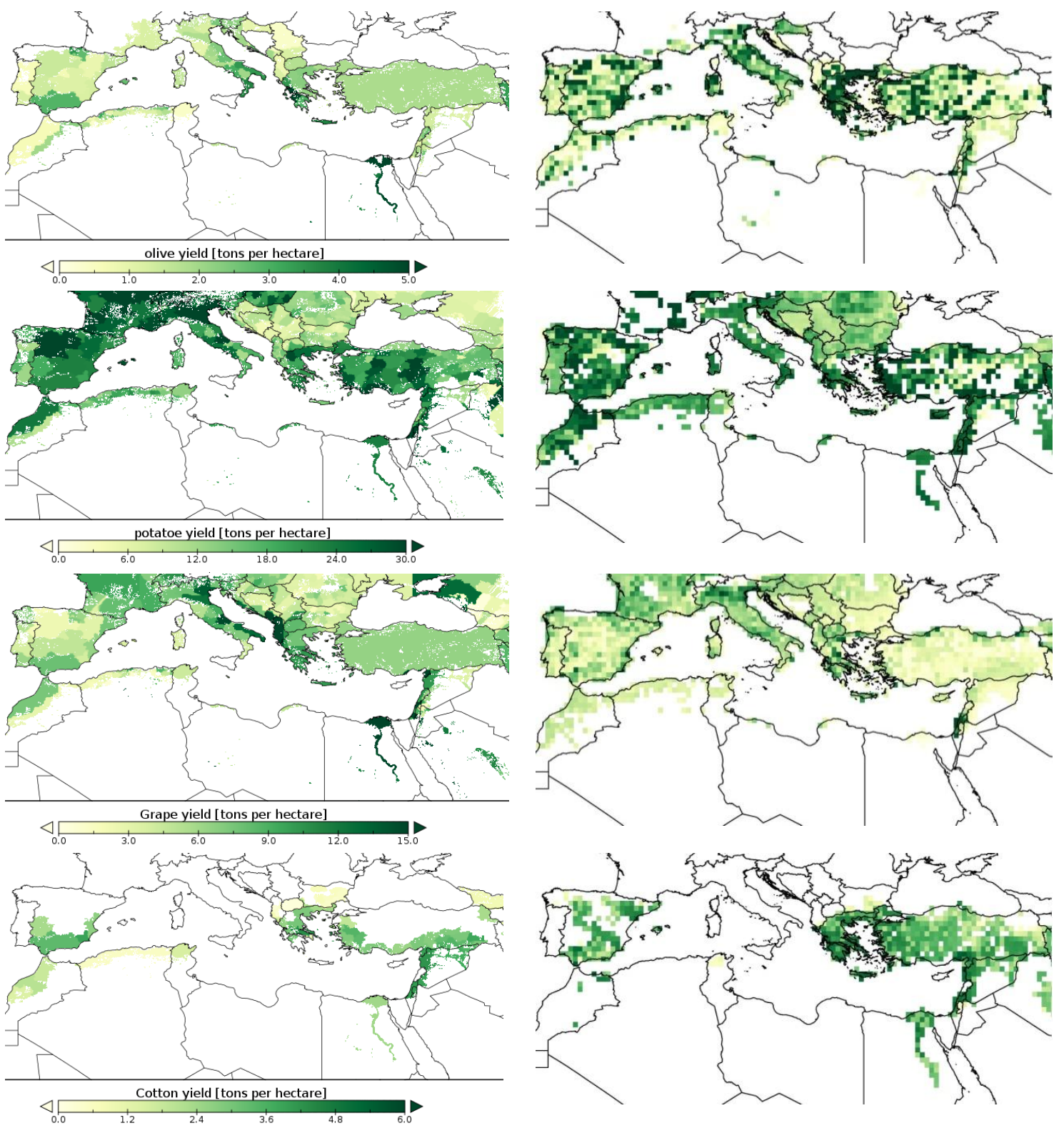

1

2 Fig. S2: Comparison of LPJmL-simulated yields (right panel, average 2000-2009) with yields from 3 Monfreda et al., 2008 (left panel, values around the year 2000) for olives, potatoes, grapes and 4 cotton. 


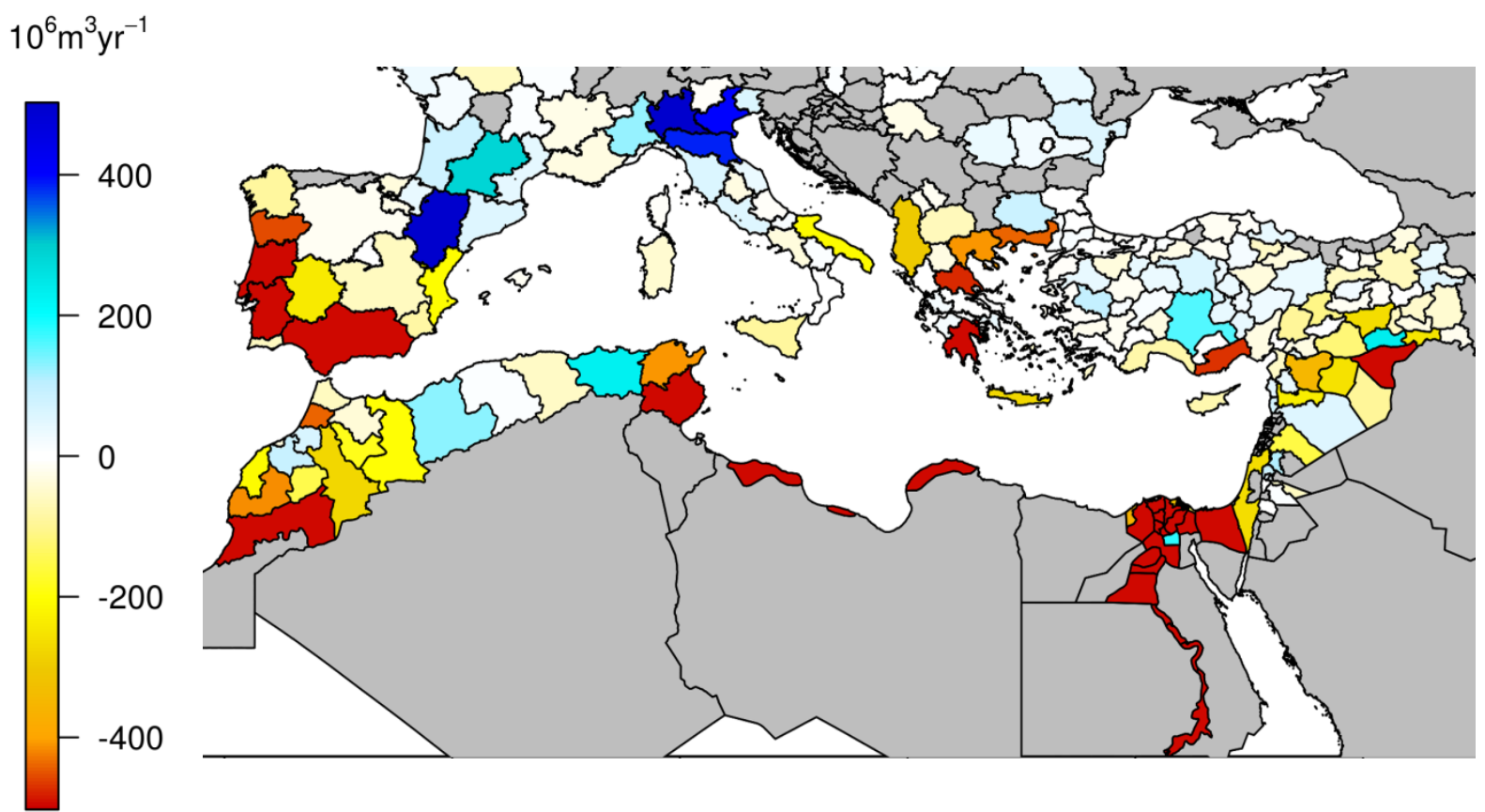

1

2 Fig. S3: Comparison of irrigation consumptive water use from Siebert et al. (2010) and net irrigation 3 water requirements computed in this study. Negative (positive) values indicate higher (lower) values 4 in their study. 